\title{
Review
}

\section{Immunohistochemical Characteristics of Odontogenic Neoplasms and Their Physiological Counterparts}

\author{
Keisuke Nakano'), Hitoshi Nagatsuka'), Hidetsugu Tsujigiwa ${ }^{3)}$, Mehmet Gunduz ${ }^{2)}$, \\ Naoki Katase ${ }^{3)}$, Chong Huat Siar ${ }^{4)}$ and Toshiyuki Kawakami ${ }^{1)}$
}

\author{
1) Hard Tissue Pathology Unit, Matsumoto Dental University Graduate School of Oral Medicine, Shiojiri, 399-0781 Japan \\ ${ }^{2)}$ Department of Oral Pathology and Medicine, Okayama University Graduate School of Medicine, Dentistry and Pharmaceutical \\ Sciences, Okayama, 700-8525 Japan \\ 3) Department of Oral pathology and Medicine, Okayama University Graduate School of Medicine, Dentistry and Pharmaceutical \\ Sciences, Okayama, 700-8525 Japan \\ 4) Department of Oral Pathology, Faculty of Dentistry, University of Malaya, 50603 Kuala Lumpur, Malaysia \\ (Accepted for publication, October 1, 2008)
}

\begin{abstract}
Development of the tooth is a complex and fascinating set of processes which require a sequential integration of numerous biological steps. For dental doctors, interest is particularly high, because the tooth is mainly composed of surface ectodermal epithelium and neural crest derived neuroectodermal mesenchyma, and formed by epithelial-mesenchymal interactions. There are many different types of odontogenic neoplasms. In general, proliferation, development and cytological differentiation of the neoplastic cells reflect the normal physiological development of the outbreak mother cells and/or tissues. There would appear to be a relationship between the cytological differentiation of odontogenic neoplastic cells and the physiological development and differentiation of tooth germ. We describe some morphogenesis regulation factors, such as Notch signaling, in the odontogenic neoplastic cells, in both well-differentiated and poorly-differentiated neoplasms. Our results suggest that these factors play some role in cytological differentiation or acquisition of tissue-specific characteristics in neoplastic cells.
\end{abstract}

Key words: Odontogenic neoplasm, Tooth, Development, Immunohistochemistry

\section{Introduction}

A neoplasm is an abnormal growth mass of cells and/or tissue of which exceeds and is uncoordinated with the physiological cells and/or tissues. Thus, neoplasms are said to be transformed because they continue to replicate, apparently oblivious to the regulatory influences that control physiological original cell and/ or tissue growth. The words "differentiation and anaplasia” are applied to the originating parenchymal cells that constitute the transformed neoplastic components of neoplasms. The word “differentiation" of parenchymal cells refers to the extent to which they resemble their physiological features, both morphological and functional in findings.

\section{Biology of neoplasms}

In benign neoplasms, the parenchymal cell components consist of well-differentiated cells that quietly resemble their originating mother cell and/or tissue. In benign neoplasms, mitoses are

Correspondence to: Dr Keisuke Nakano, Hard Tissue Pathology Unit, Matsumoto Dental University Graduate School of Oral Medicine, Shiojiri, 399-0781Japan, Phone \& Fax: +81-(0)263-51-2035,E-mail: keisuke1@po.mdu.ac.jp extremely rare in number and are of normal physiological features. In malignant neoplasms, they are characterized by a wide variety of parenchymal cell differentiation, from completely well- to undifferentiated. Malignant neoplasms are composed of poorly- to un-differentiated cells when said to be anaplastic. Anaplasia, a lack of differentiation, is thought to be a standard of malignancy. Anaplasia means literally to "form backward". In fact, it is well accepted that a malignant neoplasm originates from stem cells in tissues. Therefore, a failure of differentiation, rather than dedifferentiation of physiologically specialized cells, accounts for un-differentiated neoplasms. In general, the more malignant and the more un-differentiated (anaplastic) a neoplasm, the less likely it is to have specialized functional activity. The cells in benign neoplasms are almost always well-differentiated and resemble the physiological normal cells of the originating mother cells and tissue. The cells in malignant neoplasms are more or less differentiated, but some loss of differentiation is always present. The differentiation in these neoplastic lesions is controlled by, in part, the normal physiological regulation system. The regulation mechanism of specialization growth of a physiological 

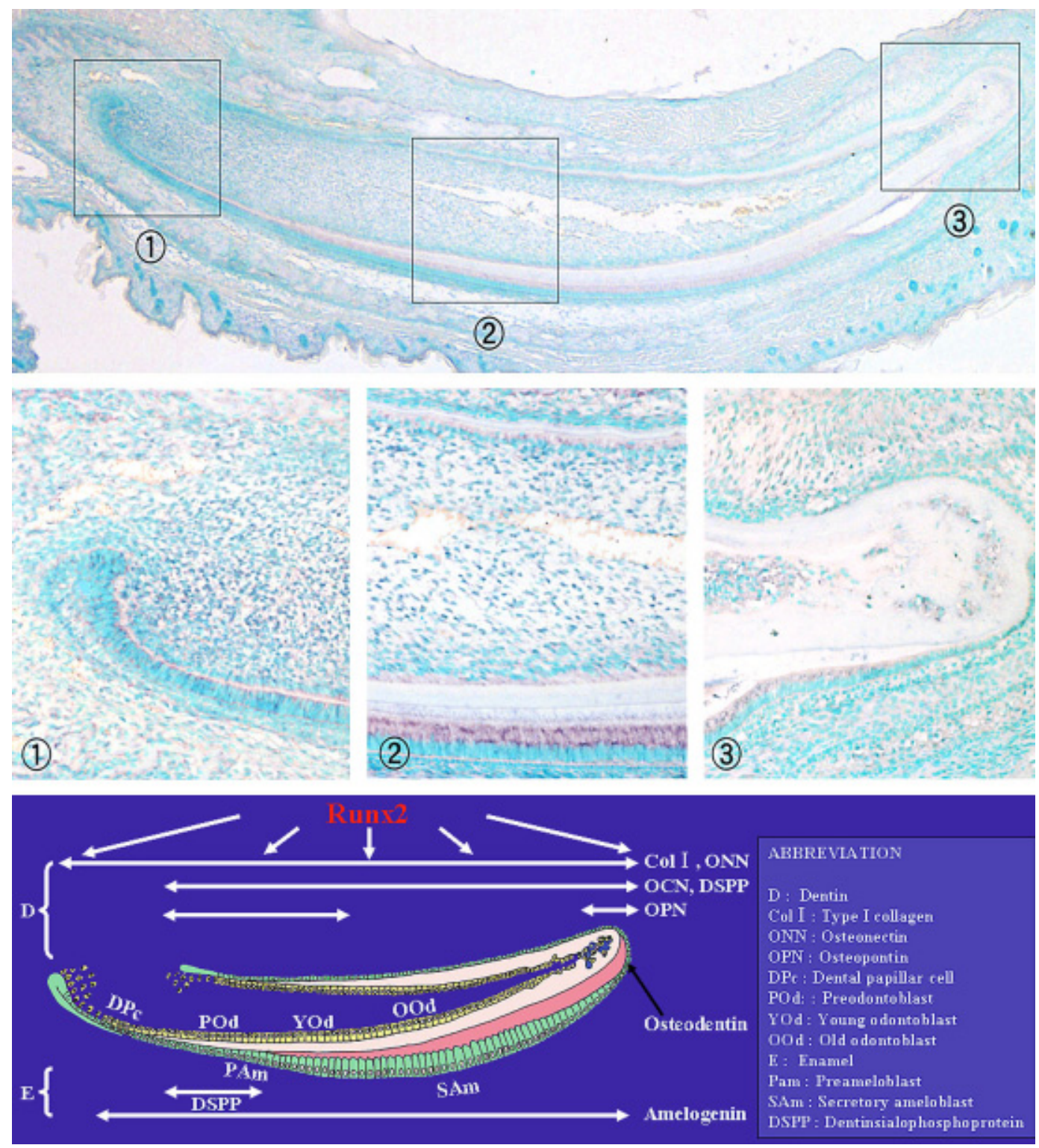

Figure 1. Runx2 regulatory control of the signaling pathway of odontogenesis. The gene expression is visible in various stages of ameloblasts (PAm, SAm, and maturation stage).

organization functions on a neoplasm, and the specialized growth of neoplasms is also regulated. Therefore, it is important to examine the normal physiological cell differentiation mechanisms for understanding the regulation system of neoplastic conditions.

\section{Developmental biology of tooth}

Teeth develop physiologically, as deciduous teeth and permanent teeth, from oral ectodermal epithelium and neural crest derived neuroectodermal mesenchymal cells ${ }^{23}$. The enamel is derived from ectoderm of the oral cavity, and all other tissues differentiate from the mesenchyme derived from mesoderm; neural crest cells are imprinted with morphogenetic information before or shortly after they migrate from the neural crest. Tooth development is initiated by the inductive influence of the neural crest mesenchyme on the covering ectoderm. Tooth development is a continuous step. However, it is usually divided into a bud stage, cap stage, and bell stage.

Odontogenesis, or tooth development, is a complex and highlyregulated process characterized by sequential epithelialmesenchymal interactions leading to tooth initiation, morphogenesis and cell-differentiation with eventual formation of enamel, dentin and cementum matrices ${ }^{18,20)}$. Osteogenesis or bone formation is also a tightly-coordinated process involving many different tissues that interact with each other via a matrixmediated inductive mechanism, and ending in the formation of a specialized tissue, bone. Both of these processes, though distinct, are closely related in that they share common signaling pathways 0 

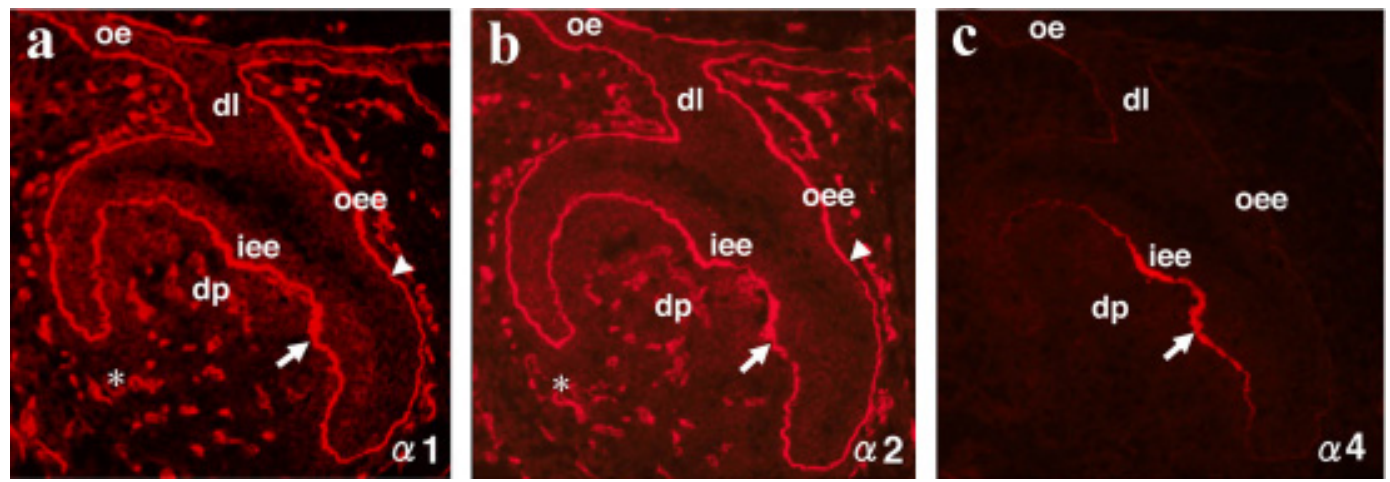

Figure 2. Distribution of $\alpha$ chains of type IV collagen in early bell stage of E15 mice. The basal membrane of inner enamel epithelium (iee, arrow) stains for $\alpha 1$ (a), $\alpha 2$ (b), and $\alpha 4$ (c) chains. oee: outer enamel epithelium, dp: dental pulp, oe: oral epithelium, dl: dental lamina, *: vascular basal membrane [quoted from Nagai N, et al. ${ }^{31}$ ]

functional differentiation of their matrix proteins. The ameloblasts and odontoblasts, which are exclusive enamel matrix-producing and dentin matrix-producing cells, respectively, share several molecular characteristics with the osteoblast, which is the bone matrix-forming cell. Runx2, a transcription factor, is essential for osteoblast differentiation (Figure 1). In the course of odontogensis, the Runx2-knockout mice experiments results suggest the following: Runx2 is associated with morphogenesis of teeth and matrix protein gene expression ${ }^{33}$. Next, compared to the incisor tooth germ, the molar tooth germ is more strongly subjected to control by Runx2, suggesting the presence of factors involved in odontogenesis of the incisor tooth germ which are different from those present in osteoblasts. Furthermore, in Runx2knockout mice differences in expression of osteopontin and osteocarcin, matrix proteins common for teeth and bone, suggest different mechanisms of cellular differentiation or transcription regulation pathways in incisor odontoblast and bone forming cells, or osteoblasts ${ }^{34)}$.

The tooth germ basal membrane interposed between the odontogenic epithelium and mesenchyme mediates the sequential and reciprocal epithelial-mesenchymal interactions essential for morphogenesis and cell-differentiation for tooth formation (Figure 2). It composes some isoforms of type IV collagen, laminin, nidogen/entacin, heparan sulfate, proteoglycan, fibronectin, and other component molecules ${ }^{30,37,61)}$. The molecules of type IV collagen, a major framework-forming peptide of basal membrane, are heterotrimers composed of three $\alpha$ chains that exist in six genetically-distinct forms ( $\alpha 1$ to $\alpha 6$ ) and with at least three molecular forms ${ }^{41,46,47)}$. The expression patterns of type IV collagen molecular forms in tooth germ organogenesis and the marked stage-specific changes in the type IV collagen distribution during the odontogenesis are limited ${ }^{21)}$. According to the examination results using mouse developing molar tooth germ at the dental placode and bud stage in the course of odontogenesis, the basal membrane of the oral cavity epithelium expresses $\alpha 1, \alpha 2, \alpha 5$ and $\alpha 6$ chains while the gubernaculums dentis, in addition to the above
4 chains, also expresses $\alpha 4$ chain. An asymmetrical distribution of $\alpha 4, \alpha 5$ and $\alpha 6$ chains has been observed at the bud stage in the odontogenesis. At the early bell stage, the basal membrane associated with the inner enamel epithelium of molar germ expresses $\alpha 1, \alpha 2$ and $\alpha 4$ chains while the basal membrane of the outer enamel epithelium only expresses $\alpha 1$ and $\alpha 2$ chains. With the onset of dentin formation, the collagen $\alpha$ chain profile of the basal membrane of inner enamel epithelium gradually disappeared. From the bell stage, however, the gubernaculums dentis consistently expressed $\alpha 1, \alpha 2, \alpha 5$ and $\alpha 6$ chains, and this distribution pattern resembles the one of the fetal oral cavity epithelium. These features suggest that the odontogenic stageand the position-specific type IV collagen $\alpha$ subunit distribution is according to the tooth germ odontogenesis, and its changes are essential for the morphogenesis and cell-differentiation for tooth development ${ }^{7,30,58)}$.

Tenascin is an extra-cellular matrix glycoprotein which appears to regulate cell morphology ${ }^{31)}$. It is more restricted to tissue distribution than fibronectin and is able to interface with the cellbinding function of fibronecion. Tenascin is most typically expressed in epithelial-mesenchymal interactions during physiological development and in the stromal tissue of malignant neoplasms. Extracellular matrix protein is shown to play important roles in cellular growth and differentiation, in complex cell matrix interactions, in physiological organ development and in neoplastic transformation course ${ }^{31)}$.

At first, in dental lamina of the bell stage of human tooth germ, tenascin is present only on the submucosal connective tissue side, not on the dental follicle tissue side ${ }^{5}$. At this stage there are no morphological differentiations in the odontogenic epithelium on either side. Concerning fibronectin, a weak or negative localization is seen in the condensed mesenchyme surrounding the dental lamina. In the cap stage, different patterns of the distribution between tenascin and fibronectin ares evident in the human tooth germ. Strong tenascin accumulation is present in the dental papilla under the basal membrane, preodontogenic layer 

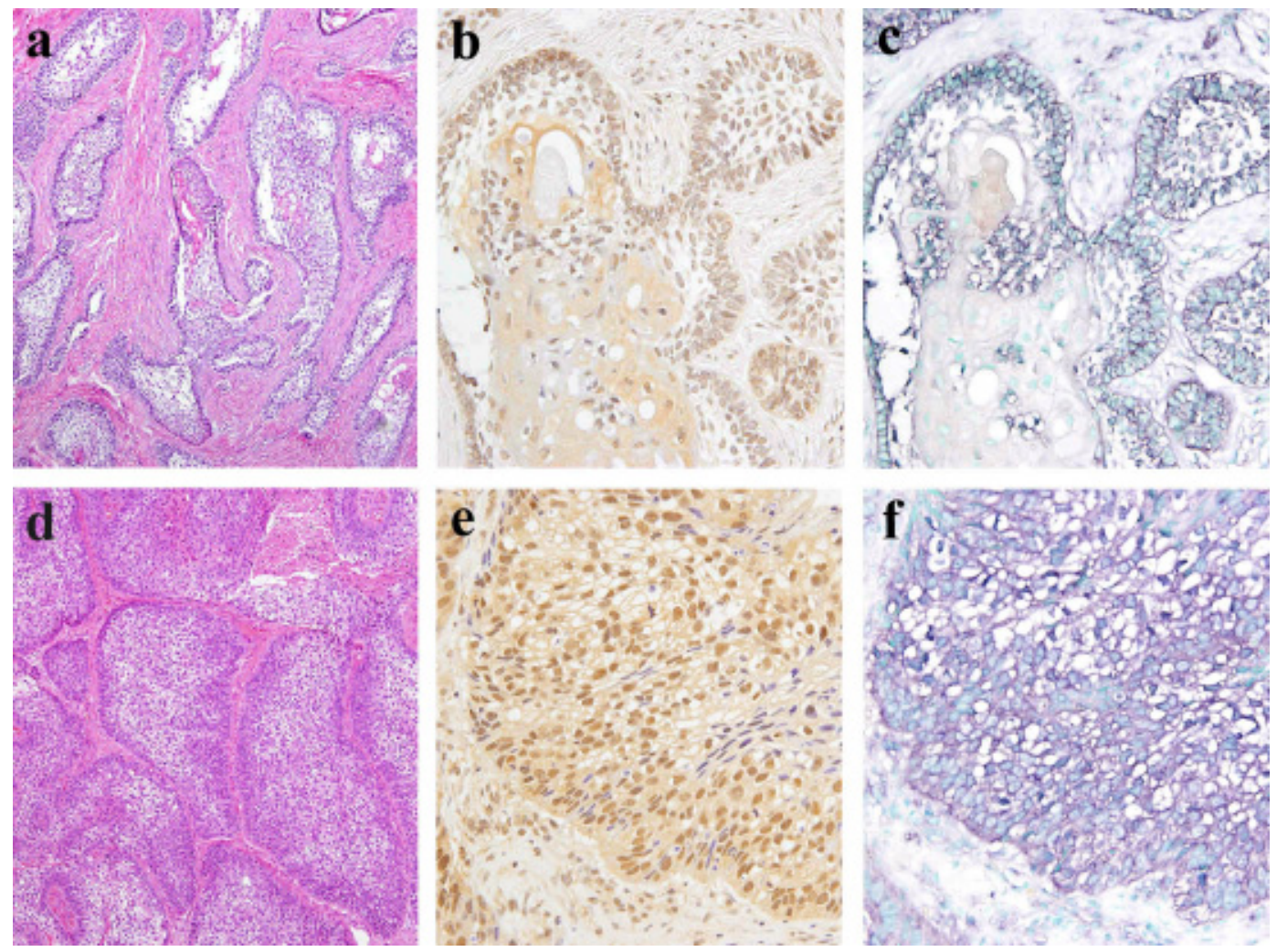

Figure 3. Proliferating follicular nests of ameloblastoma are visible (a). Immunohistochemically Notch peptides are observed in the cells at the peripheral layer (b) and gene signals are detected in the cytoplasm (c). Polyhedral neoplastic cells are visible in proliferating ameloblastic carcinoma cell nests (d). The Notch positive products are observed uniformly in these cells (e) and the gene expressions are also detected in the cytoplasm (f).

and osteogenic tissue of alveolar bone. However, tenascin is immunohistochemically negative in the dental follicle, the fibroblastic layer developing into the periodontium ${ }^{31)}$. Intense fibronectin is evident in the alveolar bone. The epithelial components of the tooth germ are imunohistochemically negative for both tenascin and fibronectin.

\section{Notch signaling in cell differentiation and development}

In general, Notch signaling plays an important role in the regulation of cell fate, morphogenesis and/or development ${ }^{4,15)}$. Regarding tooth development, there are some published data on how the expression of Notch1, 2, and 3 is regulated by epithelialmesenchymal interactions in the developing mouse tooth and associated with determination of ameloblast cell fate. Jagged1 is also expressed as a ligand of Notch in the developing tooth ${ }^{28)}$. Notch signaling is an evolutionarily-conserved cell-to-cell transmembrane interaction mechanism. Furthermore, asymmetric distribution of Notch has been observed in immature cells prior to cell division, suggesting a role in the regulation of daughter cell fate, including whether the cells remain stem cells or give rise to differentiated progeny ${ }^{36}$. Regarding odontogenesis, Notch1 is expressed in stellate reticulum cells, and Jagged1 is expressed in differentiated ameloblasts in the course of tooth development.
During tooth development, Notch expression has been associated with the differentiation of odontogenic epithelial and mesenchymal tissues. However, Notch expression is absent in epithelial cells in close contact with mesenchyme, a feature which may be important for ameloblast cell fate. These data suggest that mesenchymal tissue negatively regulates Notch expression in epithelium. In other words, Notch expression is downregulated in odontogenic epithelium juxtaposed to mesenchyme, indicating that odontogenic epithelium needs a mesenchyme-derived signal to maintain the down regulation of Notch ${ }^{28)}$.

\section{Cell differentiation in pathological conditions}

In the oral and craniofacial region, there are many types of neoplasms and pathological conditions, such as odontogenic neoplasms, bone and cartilage neoplasms, pathological bone and cartilage formation and/or proliferation. Odonotogenic neoplasms consist of a plural number of blastoderms. The neoplastic cell differentiation process is complicated, and it is thought that the cell differentiation and growth pattern are copied from a physiological system of the odontogenesis.

\section{Odontogenic neoplasms}

Ameloblastoma is classified as a benign, locally-infiltrative 
Keisuke Nakano et al.: Immunohistochemistry of Odontogenic Neoplasms

odontogenic neoplasm, which is composed of proliferating odontogenic epithelial nests within a fibrous stromal tissue. Some variants have been sub-classified as follows: solid/multicystic, extraosseous/peripheral, desmoplastic, and unicystic ${ }^{10,22,24)}$. Furthermore, other variants have been reported in the literature, and these include acanthomatous, ghost cell, and vacuolated or clear cell types ${ }^{40,44,49-51,59)}$. Odontogenesis is a complex biological process, and this process is directly reflected in the development of odontogenic neoplasms ${ }^{14,19)}$, especially ameloblastomas ${ }^{16,17)}$. It is thought that the above-mentioned variants are due to the developmental complex system ${ }^{45)}$.

\section{Notch signaling in ameloblastomas and ameloblastic carcinomas}

Regarding Notch signaling, the focus of our attention is on examining Notch1 and Jagged1 peptide expression, as well as their genes, in ameloblastomas and ameloblastic carcinomas (Figure 3). The speculation on their possible roles is in cytological differentiation and proliferation of ameloblastomas. In one examined case of ameloblastoma, histopathologically, the main specimens showed follicular nests consisting of islands of odontogenic epithelium within a fibrous stroma. Cells of the peripheral layer of these islands were columnar, with hyperchromatic nuclei, and lined up in a palisade fashion, whereas the central cells were stellate reticulum-like. Their cytoplasms were generally vacuolated. Some nests showed central cyst formation. In small parts, the odontogenic epithelium exhibited focal basal palisading. Furthermore, occasionally a large number of nests underwent squamous metaplasia with keratinizing pearl formation. In general, degeneration of the parenchymal cells and cyst formation occurred in these ameloblastoma nests. According to the immunohistochemistry examination results ${ }^{25,26,38,39)}$, NICDpositive products were detected in most proliferating odontogenic epithelial nests of ameloblastomas by IHC. The positive reactions existed in the cytoplasm and/or nucleus. Strong reactions were seen in the pre-ameloblast-like cells or some localized cells within the nests. In some ameloblastoma nests, there were no positive reactions to NICD. Jagged1 positive reactions were also observed in the cytoplasms of same cell types in the ameloblastoma nests. Strong reactions existed at the peripheral layers. The pattern of distribution and the intensity of expression of Jagged1 were closely similar to the pattern and intensity seen in NICD. Notch1 gene signaling was localized in the cytoplasm of IHC-positive neoplastic cells. These mRNA positive signals showed variable labeling intensity. Jagged1 mRNA signals were also detected in the cytoplasm of ameloblastoma cells, and the strength pattern was nearly the same as that of Notch1. These mRNA signal expressions were not consistent with those of the transcription factor peptides. Histopathologically, the follicular type of ameloblastoma is the most common, consisting of proliferating odontogenic epithelial islands and nests in the fibrous stromal tissues. Cellular modifications, such as squamous metaplasia, keratin pearl formation, parenchyma cell degeneration and cystic changes, may also occur. Morphogenesis is a complex biological process, and this process directly reflects the development and proliferation of neoplasms. Regarding the proliferation of ameloblastomas, some morphogenesis factors are overlyexpressed in ameloblastoma tissues in comparison with tooth germs. According to the analysis of gene expression in ameloblastomas and human fetal tooth germs using a cDNA microarray, some results have been published. Aanalysis included tumor-necrosis-factor-receptor-1 (TNFRSF-1), sonic hedgehog (SHH), Cadherins 12 and 13 (CDH 12 and 13), and transforming growth-factor-1 (TGF- $\beta 1$ ), the gene expression profile identified candidate genes that might be involved in the origination of ameloblastoma, as well as several genes previously unidentified in relation to human tooth development. The expression of SHH signaling in ameloblastomas, in comparison with human tooth germs, was also detected. The literature concluded that the $\mathrm{SHH}$ signaling might play a role in epithelial-mesenchymal interactions and cell proliferation in the growth of ameloblastomas ${ }^{27)}$.

On the transcription factors Notch1 and Jagged1 in ameloblastoma, the results demonstrate that Notch1 (NICD) and Jagged 1 are both detected by IHC, and their expression patterns are very similar ${ }^{62)}$. This phenomenon means that Notch signaling is activated in the neoplastic epithelium of ameloblastoma. It is likely that the signaling plays the role of daughter cell fate regulation. Positive Notch1 reactions suggest that proliferation and cytological differentiation are probably occurring in these neoplastic cells. This explains the variation in the strength of these signals and their distribution patterns in the ameloblastoma cell nests. Furthermore, the mRNA of Notch1 and Jagged are also expressed in the ameloblastoma cells, as determined by ISH. These mRNA signal expressions are consistent with those of the transcription factor peptides. The examination of larger case series of ameloblastoma and other odontogenic epithelial neoplasms, including epithelial, mesenchymal, benign and malignant entities such as calcifying epithelial odontogenic tumor, adenomatoid odontogenic tumor, keratocystic odontogenic tumor, ameloblastic fibroma, odontoma, and odontogenic carcinoma, would help to elucidate further the role of these genes in odontogenic tumorgenesis. The results suggest that Notch signaling plays a role in cytological differentiation or acquisition of tissue-specific characteristics in these neoplastic cells of ameloblastomas ${ }^{50)}$.

Histopathologically, in ameloblastic carcinoma, proliferating polyhedral neoplastic cells show strong cellular atypia, such as mitosis and pleomorphism, especially in peripheral layers of the nests. NICD-positive products are observed in most proliferating nests of benign ameloblastoma by IHC, and strong reactions are seen in the cells at the peripheral layer of the nests. In the case of ameloblastic carcinomas, positive products have also been detected, and strong reactions uniformly observed. The positive reactions are comparatively weaker in benign than in malignant 

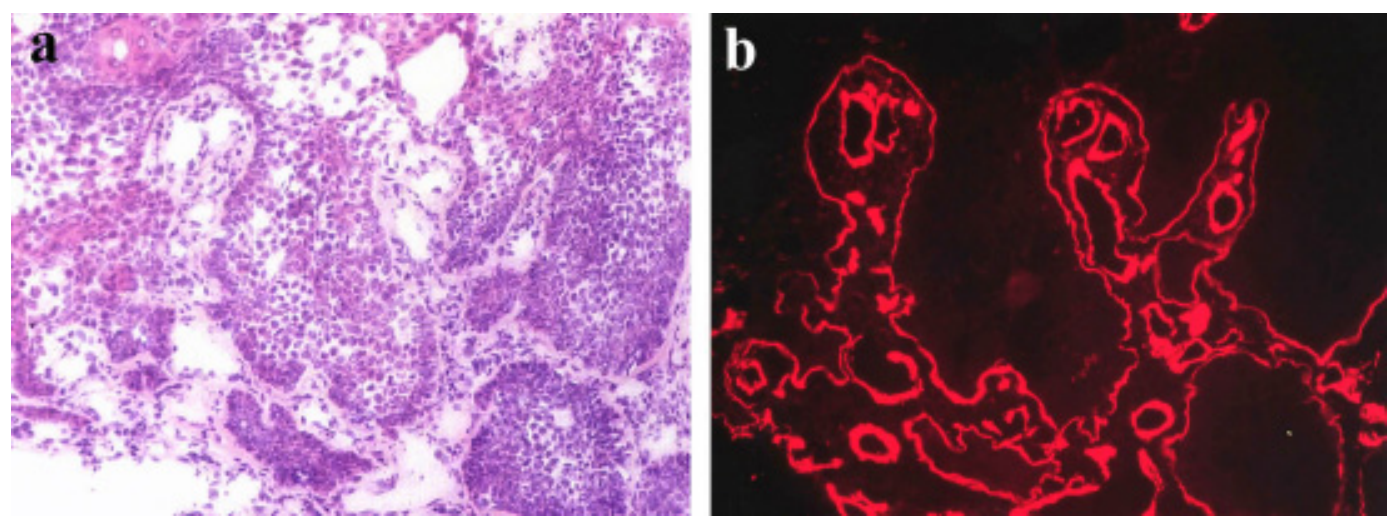

Figure 4: Histopathological feature of examined plexiform ameloblastoma (a) and immunofluoresence localization of type IV collagen $\alpha 1$ chain in the basal membrane zone (b).[quoted from Nakano K, et al. ${ }^{36}$ ]
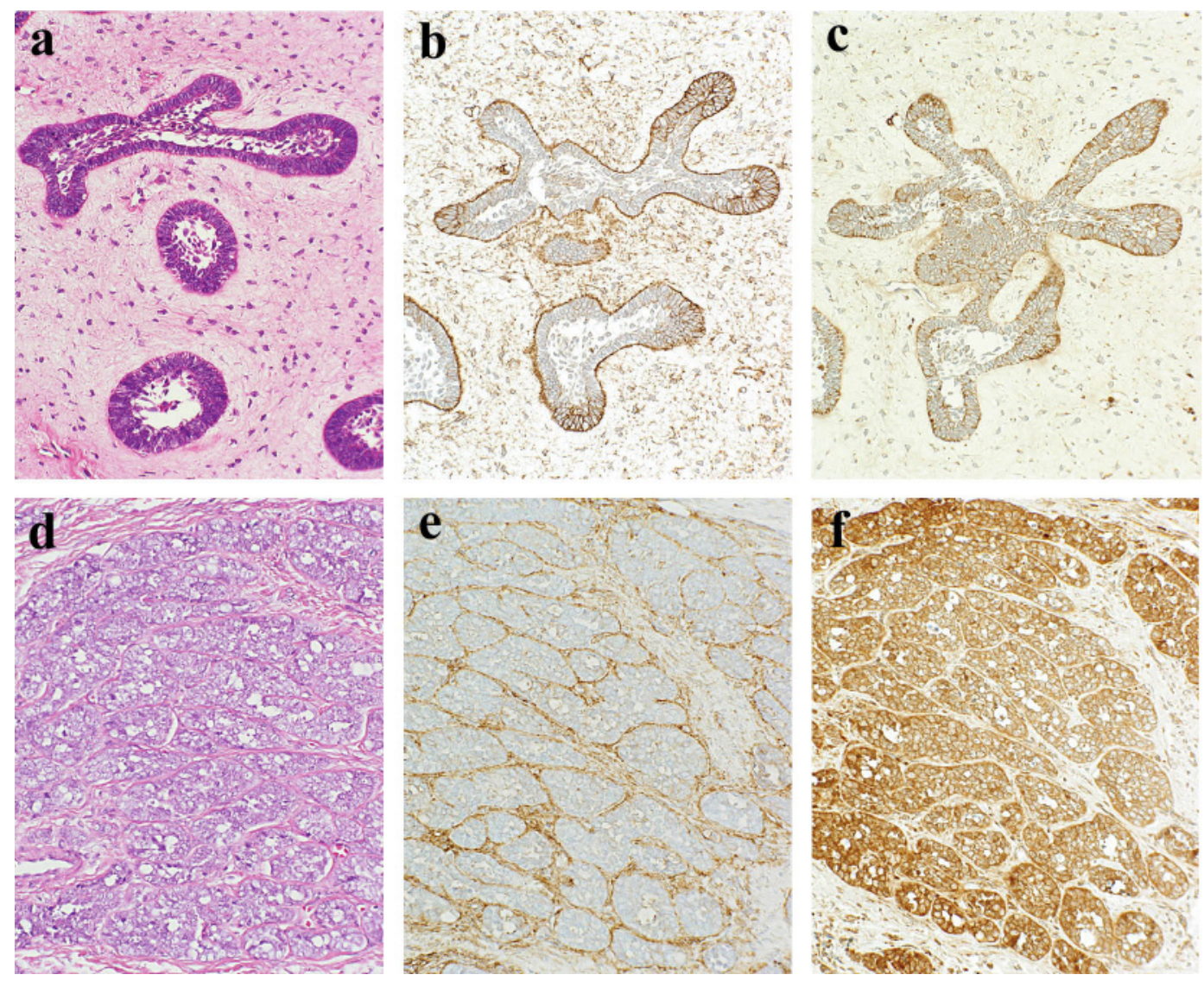

Figure 5. Ameloblastic epithelial islands within scattered dental papilla-like ectomesenchymal tissue (a). HS and heparanase immunohistochemical reactivity accentuates the cellular processes of dental papilla-like cells (b and c). Note the clear confinement and continuity of basal membrane despite the prominent localization of heparanase (b). Histopathologically, cancer cell nests of ameloblastic carcinoma increase nuclear-cytoplasmic ratio and prominent nuclei (d). HS is not detected in the cancer cells but is strongly localized in the stromal tissues adjacent to cancer nests (e). Intense and diffuse heparanase expression is observed in all cancer cells (f) in contrast to the strong staining limited in the basal cells of benign ameloblastoma (c). [quoted from Han PP, et al. $\left.{ }^{12)}\right]$

tumors. In both benign and malignant cases, gene (mRNA) expressions have been detected in the cytoplasms of IHC-positive cells by ISH. In general, Notch signaling is responsible for cytological regulation of cell fate, morphogenesis and/or development. In examinations conducted by the present authors,
IHC and ISH examination results have suggested that Notch signaling plays some role in cytological differentiation or acquisition of tissue-specific characteristics in neoplastic cells of tooth enamel organ-derived neoplasms, including benign and malignant neoplasms, ameloblastoma and ameloblastic carcinoma. 
Keisuke Nakano et al.: Immunohistochemistry of Odontogenic Neoplasms

Histopathologically, follicular nests proliferate in the fibrous connective tissue in benign neoplasms. In some nests, parenchymal cyst formations or squamous metaplasia are evident. The histopathological features of the malignant neoplasms are as follows: proliferating polyhedral neoplastic cells show strong cellular atypia, such as mitosis and pleomorphism, especially in peripheral layers of the nests. Notch intra-cellar domain positive products are observed in most proliferating nests of benign neoplasms by immunohistochemistry. Strong reactions are seen in the cells at the peripheral layer of the nests. In malignant ones, positive products are also detected, and strong reactions uniformly observed. The positive reactions in benign neoplasms are comparatively weaker than in malignant ones. In both the benign and malignant cases, the gene expressions are detected in the cytoplasm of immunohistochemistry-positive cells by ISH. Our examination results suggest that Notch signaling plays some role in cytological differentiation or acquisition of tissue specific characteristics in neoplastic cells. Furthermore, there would appear to be a relationship between the cytological differentiation in the oral and craniofacial neoplastic cells and the physiological development and differentiation of their originating mother cells and tissues of the oral and craniofacial region.

\section{Cell differentiation in odontogenic neoplasms}

Amelogenin is a typical enamel matrix protein. The expression pattern of amelogenin genes (AMGX, AMGY) has not yet been identified in ameloblastomas. In surgical materials, amelogenin gene is expressed in all ameloblastoma cells. The mRNA of AMGY expression increases, although that of AMGX does not. This is an interesting feature in physiological normal male tooth development, in which the expression of AMGY is considerably lower than that of AMGX. This finding suggests that epigenetic change of sex chromosomes may have some correlation with tumorigenesis of ameloblastoma ${ }^{60)}$.

Regarding the collagen subunits of basal membrane components of oral neoplasms, there are some published data ${ }^{1,11,53,54,55)}$. Regarding ameloblastomas, co-expression of type IV collagen $\alpha 1$ and $\alpha 2$ chains appears as thin lines with limited areas of discontinuity along the basal membrane of neoplastic cell nests ${ }^{35,36)}$. The expression staining is strong and in a linear continuous manner, in the periphery of the nests of the desmoplastic types. In the neoplasms, $\alpha 5$ and $\alpha 6$ chains are colocalized as continuous linear patterns demonstrating the tumoral nests from the surrounding connective tissue stroma. These collagen subunits also appear as random intracellular staining of the neoplastic cell nests ${ }^{8,9}$. There is no remarkable differentiation of the distribution pattern among the tumor growth patterns and various cellular subtypes within ameloblastomas (Figure 4). Distribution of $\alpha$ subunit of collagen in the basal membrane of ameloblastic fibromas is uniformly demonstrated in its pattern. Subunits of $\alpha 1 / \mathrm{a} 2, \alpha 4$ and $\alpha 5 / \alpha 6$ are distributed as liner continuous patterns that compartmentalize the neoplastic epithelial cell nests, islands and strands from the surrounding dental papilla-like ectomesenchymal cell proliferation. These $\alpha$ subunits of collagen are randomly expressed in the periphery preameloblast-like and central stellate reticulum-like cells. In adenomatoid odontogenic tumors, subunits of $\alpha 1 / \alpha 2$ and $\alpha 5 / a 6$ are strongly expressed at the area of interface between tumor cells and stromal tissues, especially in the cribriform regions. Faint to non-positive expression of these collagen molecules is detected in the basal regions of conglomerated masses of solid epithelial whorls/ rosettes/nests and duct-like structures. There are intensely-positive reactions to the amorphous deposits; however, there is little or no reaction to the mineralized bodies. In malignant neoplasms, at first in ameloblastic fibro-odontosarcomas, $\alpha 1 / \alpha 2$ and $\alpha 4$ chains demonstrate moderate intensity along with the periphery of the epithelial components, while $\alpha 5 / \alpha 6$ chains are strongly codistributed as continuous linear patterns demarcating the benign neoplastic cell nests from the surrounding sarcoma tissues. In the inductive dental hard tissue regions, no reactivity is found. In the malignant neoplasm type of ameloblastic carcinomas, collagen IV $\alpha$ chains demonstrate an irregular and disrupted expression pattern with specific loss of $\alpha 1 / \alpha 2$ chains. In those regions containing poorly-differentiated neoplasm cell nests, there is complete disappearance of $\alpha$ chain subunits. In $\alpha 5 / \alpha 6$ subunits, there is a discontinuous and fragmented pattern. In primary intraosseous carcinomas, the expression pattern is similar to that of ameloblastic carcinomas. From the above-mentioned findings for cell differentiation in various benign and malignant odontogenic neoplasms, the basal membrane pattern of neoplastic epithelial cell nests yields three features: (1) the basal membrane of benign and malignant odontogenic neoplasms has distinct $\alpha$ chain subunits of collagen type IV; (2) modifications in the relative abundance of collagen type IV $\alpha$ chains in basal membrane of odontogenic neoplasms probably represent a host protective response; and (3) early specific loss of $\alpha 1 / \alpha 2$ chains proceeds the loss affecting $\alpha 5 / \alpha 6$ chains during odontogenic neoplasm progression. Therefore, these results suggest that modification and remodeling of basal membrane collagen type IV $\alpha$ chains are dynamic processes crucial for odontogenic neoplastic cell growth and progression ${ }^{6,36)}$.

Heparan sulphate (HS) and heparanase appearing in the odontogenic neoplasms are interesting molecules for these neoplastic transdifferentiations ${ }^{12,13,43)}$. HS proteoglycans (HSPG) constitute a group of ubiquitous extracellular matrix macromolecules and are composed of a core protein and covalently linked HS sugar chains (Figure 5). Although HSPG plays critical functions in cell-to-cell and cell-to-matrix interactions through core proteins, their HS chains confer most of their biological functions ${ }^{2)}$. The negativel-charged HS chains can bind and sequester numerous heparin/HS binding molecules, including growth factors, cytokines and cell adhesion molecules. HS chains 
also take part in the important cellar events conferred by these tethered molecules and have an influence on various developmental and pathological processes, such as neoplastic transformation, its local invasiveness and transdifferentiation ${ }^{2,3)}$.

In the examination results on ameloblastomas, HS is clearly evident on the cell surface of peripheral basal cells and also in the intercellular region of some parabasal cells, while it is not present in the central stellate cells of ameloblastoma nests. Heparanase is expressed in peripheral epithelial cells of ameloblastoma nests. The strong expression is localized at the budding region of the strands mainly in the invasive fronts in histopathological specimens. In adenomatoid odontogenic tumors, the strongly localized and limited expression is present on the surfaces of dark cuboidal cells surrounding the whorls and solid tumor cell nests. HS is also evident in luminar surfaces of some duct-like structures. In the lumen and/or duct-like structures, eosinophilic materials are HS positive. The periphery of immature calcified materials are positive to both HS and heparanase; however, completely calcified materials are negative.

In ameloblastic fibromas, as one of typical epithelial and mesenchymal mixed odontogenic neoplasms, HS exists in nearly the same location as in ameloblastomas. HS is evident in the basal membrane, but is more defined. HS is also present in the ectomesenchymal cells, unlike the stromal cells in ameloblastomas. HS and heparanase are diffusely present both in epithelial and mesenchymal tissue of the neoplasms. In some parts, heparanase exists focally in nuclei of mesenchymal cells. In ameloblastic carcinomas as malignant odontogenic neoplasms, neoplastic cells are absent of HS in contrary in benign neoplasms, such as ameloblastomas. Instead, HS clearly and strongly is present in the stromal tissues, especially in the intercellular matrices within the vicinity of neoplastic cell nests. Regarding heparanase activity, positive reactions are intense and diffuse, and occur in intracellular spaces. The above-mentioned findings are compared with the data of physiological tooth development, in both experimental animals and human materials. Furthermore, when the results are examined using various types of oral squamous cell carcinoma, there are some differences between the types of histological and clinical malignancy grades. In summary, the general localization of HS and the heparanase activity in odontogenic neoplasms are temporally regulated in relation to cellular growth and function. Furthermore, heparanase overexpression is reported to promote hair follicle morphogenesis and its growth ${ }^{13,62)}$. Both hair follicular morphogenesis and odontogenesis are governed by similar growth factors and signaling pathways. Heparanase may also have physiological function in tooth development through local modulation and release of HS-bound growth factors. Taken together, the facts suggest that heparanase may have physiological function in tooth development, and the increase in heparanase expression maybe an important initiating factor for odontogenic neoplastic transformation. The stromal HS sugar molecule localization and heparanase over-expression may represent the malignant progression of ameloblastoma to ameloblastic carcinoma ${ }^{32)}$.

In odontogenic neoplasms, both benign and malignant, the immunohistochemical distribution of tenascin and fibronectin is compared with that in human tooth germs ${ }^{56,57)}$. In ameloblastomas, the extracellular matrix components of the stromal tissue of ameloblastomas exhibit considerable variety: dense and loose connective tissues, hyalinization regions, and stromal cystic spaces. In the hyalinised stroma, tenascin and fibronectin exhibit both positive and negative reactions. In cystic spaces, positive reactions of tenascin and fibronectin are seen. In follicular type of ameloblastomas, the basal membrane region reacts irregularly positive to tenascin. The fibronectin reactivity exhibits uniformly and weakly positive in the dense connective tissue of the stromal region in the follicular ameloblastomas. A partial accumulation of tenascin is found in the basal membrane. The tenascin-positive basal membrane shows fuzzy fibrillar materials, whereas the loose or myxomatous tissues of stromal region of follicular type of ameloblatoma exhibit no reaction to fibronectin. Regarding malignant odontogenic neoplasms, the data on ameloblastic carcinomas are as follows. The stromal tissue and basal membrane of ameloblastic carcinomas show an irregular and strong immunohistochemical-positive reaction to tenascin. In the epithelial cell islands of ameloblastic carcinomas, a scattered or granular positive reaction is evident. The connective stromal tissue of ameloblatic carcinomas shows an irregular and strong reaction to fibronectin. According to the localization pattern of tenascin and fibronectin in the varied types of odontogenic neoplasms, such as benign and malignant, the dental follicle of the tooth germ lacks tenascin but has fibronectin. The osteogenic tissues generally contain both tenascin and fibronectin. The ameloblastic fibromas show positive or negative distributions in the stromal tissues, which suggest a differentiation to the papilla of the tooth germ. It is also suggests that the stromal tissue cells of ameloblastic fibroma differentiate to dental follicular tissues. The relative distribution of menisci and fibronectin can be a marker in histological diagnosis of periodontal and osteogenic fibrous tissues. Furthermore, the findings also suggest that fibronectin and tenascin may be used as markers in cell differentiation of epithelial-mesenchymal interactions during tooth development and in odontogenic neoplasm for trans-differentiation ${ }^{31)}$.

Cytokeratins with intermediate filaments characteristic of epithelial cells are very stable and range in weight from 40 to $67 \mathrm{kD}^{33)}$. Regarding the distribution patterns of cytokeratins in some types of ameloblastomas, such as follicular ameloblastomas, the peripheral columnar cells resembling preameloblasts react positively for NSE-K (52.5kD) and 19-K (40kD) in a linear pattern along the basal membrane, but not for the markers of squamous cells, SE-K (56, 56.5, 58 and 68kD). The reaction pattern of NSE-K and $19 \mathrm{~K}$ shows a frame-like structure in cytoplasm in 
Keisuke Nakano et al.: Immunohistochemistry of Odontogenic Neoplasms

peripheral columnar cells, while the central stellate reticulumlike cells show an immunohistochemical reaction with all markers of the cytketain SE-K, NSE-K and 19-K throughout the cytoplasm. In plexiform ameloblastomas, both the central spindle and peripheral cuboidal cells demonstrate a positive reaction with SE-K and 19-K, but not with NSE-K. Regarding the oral mucosa and developing tooth germ, there are differences for immunohistochemical reactivities of cytokeratins between the fetal oral mucosa and adult gingiva. The cells of both the fetal oral mucosa and adult gingiva demonstrate positive reactions to SE-K, and negative reactions to NSE-K. However, positive reactivity to $19-\mathrm{K}$ is noted only in cells of the fetal mucosa and Merkel cells in adult gingiva. The dental lamina connecting with the basal cells of the oral mucosa have all these cytokeratins, especially 19-K. In the enamel organ, however, the immunoreactivity for SE-K, a maker of squamous differentiation, is different in each cell layer: positive in the outer enamel epithelium; slightly positive to negative in the stellate reticulum and stratum intermedium; and negative in the columnar inner enamel epithelium. NSE-K and 19-K are evident in all types of cells that compose the enamel organ proper. The inner columnar enamel epithelium especially expresses a diffuse positive reaction for NSE-K and 19-K throughout the cytoplasms. These features suggest that the characteristics of the cells of the plexiform ameloblastoma are similar to the fetal oral epithelium not odontogenic epithelium. This later feature suggests that the different expression patterns of cytokeratin in ameloblastoma depend on the follicular or plexiform types. As a coincidence of cytokeratin and functional pattern is not noted among the ameloblastomas and tooth germs, these data suggest that the columnar cells of the follicular ameloblastoma have little resemblance to ameloblast-like cells in cytokeratin structure or in cellular functions ${ }^{33)}$.

\section{Conclusion}

Tooth development is a complex and fascinating set of processes which require a sequential integration of numerous biological steps, and the differentiation in cells of odontogenic neoplasms is also a complex and fascinating compound process. Therefore, there are many different types of odontogenic neoplasms. It is known that proliferation, development and cytologicaldifferentiation of the neoplastic cells reflect the normal physiological development of the outbreak mother cells and/or tissues. Some cell-differentiation, development and proliferation factors may also play roles in the neoplastic cells, and therefore, their behavior is closely related in cytological differentiation and clinical behavior and/or grade.

\section{Acknowledgments}

This research was supported in part by a Grant-in Aid for Scientific Research (C) (20592349) from the Japan Society for the Promotion of Science. The authors thank Professor DM
Carlson, Matsumoto Dental University, for his critical reading of the manuscript.The authors wishes to give special thanks to Professor Emeritus Noriyuki Nagai, Okayama University, for preparation of the manuscript.

\section{References}

1. Barsky SH, Siegal GP, Jannotta F and Liotta LA. Loss of basement membrane components by invasive tumors but not by their benign counterparts. Lab Invest 49: 140-147, 1983

2. Bernfield M, Gotte M, Park PW, Reizes O, Fitzgerald ML, Lincecum J and Zako M. Functions of cell surface heparin sulfate proteoglycans. Ann Rev Biochem 68: 729-777, 1999

3. Blackhall FH, Merry CL, Davies EJ and Javson GC. Heparan sulfate proteoglycans and cancer. Br J Cancer 85: 1094-1098, 2001

4. Blaumueller CM, Qi H, Zagouras P, and Artavanis-Tsakonas S. Intracellular cleavage of Notch leads to a heterodimeric receptor on the plasma membrane. Cell 90: 281-291, 1997

5. Butler WT and Ritchie H. The nature and functional significance of dentin extracellular matrix proteins. Int J Dev Biol 39: 169-179, 1995

6. Dehan P, Waltregny D, Baschin A, Noel A, Castronovo V, Tryggvason K, Leval DJ and Foidart JM. Loss of type IV collagen alpha 5 abd alpha 6 chains in human invasive prostate carcinomas. Am J Patholo 151: 1097-1104, 1997

7. D’errico JA, Macneil RL, Takata T, Berry J, Strayorn C and Somerman MJ. Expression of bone associated makers by tooth root lining cells, in situ and in vitro. Bone 20: 117-126, 1997

8. Fujii H, Nagatsuka H, Lee YJ, Shinnou T, Tamamura R, Xiao J, Naitou I, Sado G, Nakagawa T and Nagai N. Differential expression of type IV collagen alpha 1 to 6 chains in basement membrane of human tooth germ and odontogenic tumors. J Hard Tissue Biol 13: 103-109, 2004

9. Fujii H, Nagatsuka H, Lee YJ, Shinnou T, Tamamura R, Xiao J, Naitou I, Sado G, Nakagawa T and Nagai N. Differential expression of type IV collagen alpha 1 to alpha 6 chains in basement membrane of malignant odontogenic tumors. J Hard Tissue Biol 13: 111-115, 2004

10. Gardner DG, Heikinheimo K, Shear M, Philipsen HP and Coleman H. Ameloblastomas. In: Banes L, Eveson JW, Reichart P, Sidransky D ed. World Health Organization Classification of Tumours Pathology and Genetics of Head and Neck Tumours. IARC Press, Lyon, 296-300, 2005

11. Han PP, Tamamura R, Katase N, Fujii E, Okauchi M, Jin T, Xiao J, Siar CH and Nagatsuka H. Differential distribution of type IV collagen $\alpha 1$ to $\alpha 6$ chains suggests distinct molecular between the epithelial and mesenchymal components of benign odontogenic tumors. J Hard Tissue Biol 15: 45-53, 2006 
Tamamura R, Katase N, Nakajima M, Naomoto Y and Nagai $\mathrm{N}$. Immunodetection of heparin sulphate and heparanase molecules in benign and malignant odontogenic tumors. Oral Med Pathol 11: 49-54, 2006

13. Han PP, Nagatsuka H, Tamamura R, Katase N, Bernard LM, Hu H, Takagi S, Ishida S, Nakano K, Kawakami T and Nagai $\mathrm{N}$. Role of Heparanase in the release of heparin sulphate binding growth facvtors in odontogenic tumors. J Hard Tissue Biol 16: 23-30, 2007

14. Han PP, Nagatsuka H, Siar CH, Tsujigiwa H, Gunduz M, Tamamura R, Borkosky SS, Katase N, Nagai N. A pigmented calcifying cystic odontogenic tumor associated with compound odontoma: a case report and review of literature. Head Face Med 3: 35 online: http://www.head-face-med.com/ content/3/1/35, 2007

15. Harada H, Mitsuyasu T and Toyono T. Epithelial stem cells in teeth. Odontol 90: 1-6, 2002

16. Heikinheimo K, Jee KJ, Niini T, Aalto Y, Happonen RP, Leivo I and Knuutila S. Gene expression profiling of ameloblastoma and human tooth germ by means of a cDNA microarray. J Dent Res 81: 525-30, 2002

17. Heikinheimo K, Morgan PR, Happonen RP, Stenman G and Virtanen I. Distribution of extracellular matrix protein in odontogenic tumors and developing teeth. Virchows Arch 61: 101-109, 1991

18. Heikinheimo K and Salo T. Expression of basement membrane type IV collagen and type IV collagenase (MMP-2 and MMP9) in human fetal teeth. J Dent Res 74: 1226-1234. 1995

19. Heikinheimo K, Mori K, Nagatsuka H and Happonen R-P. Transforming growth factor beta (TGF-b) gene family members in developing and neoplastic odontogenic tissues. J Hard Tissue Biol 15: 1-5, 2006

20. Hina $M$, Inoue $M$, Nagatsuka $H$, Takagi $T$ and Nagai $N$. Immunohistochemical demonstration of amelogenin, type IV collagen and fibronectin in human and rat tooth germs. J Jpn Stomatol 42: 659-664, 1993

21. Hudson BG, Reeders ST and Tryggvason K. Type IV collagen: structure, gene organization, and role in human diseases. Molecular basis of Goodpasture and Alport syndromes and diffuse leiomyomatosis. J Biol Chem 15: 26033-26036, 1993

22. Inoue M, Nagatsuka, Tamamura R, Siar $\mathrm{CH}$, Tsujigiwa $\mathrm{H}$ Borkosky S, Fujii M and Setsu K. Localization of oxytalan fiber, type III collagen and BMP family in conventional and desmoplastic ameloblastoma. J Hard Tissue Biol 17: 23-30, 2008

23. Ishida O, Inomata $\mathrm{K}$ and Nagai N. Cytokeratin filaments in odontogenic epithelium of human fetus. J Jpn Stomatol 41: 19-29, 1992

24. Katase N, Nagatsuka H, Tsujigiwa H, Gunduz M, Tamamura R, Han PP, Rivera RS, Nakajima M, Naomoto Y, Nagai N. Analysis of the neoplastic nature and biological potential of sporadic and nevoid basal cell carcinoma syndrome-associated keratocystic odontogenic tumor. J Oral Pathol Med 36: 550554, 2007

25. Kawakami T, Nakano K, Siar CH, Shimizu T, Nagatsuka H and Nagai N. Detection of Notch signaling in tooth germ enamel organ-derived neoplastic cells: Immunohistochemical and in situ hybridization examinations. Microsco Microanal 13: 328-329, 2007

26. Kawakami T, Nagatsuka H, Nakano K, Shimizu T, Tsujigiwa $H$, Hasegawa $H$ and Nagai N. Cell differentiation of neoplastic cells originating in the oral and craniofacial regions. In Cell Differentiation Research Developments. pp1-30, Ivanova LV ed, Nova Science Publishers Inc, NY, 2008

27. Kumamoto H, Ohki K and Ooya K. Expression of sonic hedgehog (SHH) signaling molecules in ameloblastomas. J Oral Pathol Med 33: 185-190, 2004

28. Mitsiadis TA, Fried K and Goridis C. Reactivation of DeltaNotch signaling after injury: complementary expression pattern of ligand and receptor in dental pulp. Exp Cell Res 246: 312-318, 1995

29. Mitsiadis TA, Henrique D, Thesleff I and Lendahl U. Mouse Serrate-1 (Jagged-1): expression in the developing tooth is regulated by epithelial-mesenchymal interactions and fibroblast growth factor-4. Development 124: 1473-1483, 1997

30. Nagai N, Nakano K, Sado Y, Naito I, Gunduz M, Tsujigiwa $\mathrm{H}$, Nagatsuka H, Ninomiya Y and Siar CH. Localization of type IV collagen a1 to a6 chains in basement membrane during mouse molar germ development. Int J Dev Biol 45: 827-831, 2001

31 Nagai N, Yamachika E, Nishijima K, Inoue M, Shin HI, Suh MS, Nagatsuka H. Immunohistochemical demonstration of tenascin abnd fibronectin in odontogenic tumors and human fetal tooth germs. Oral Oncol, Eur J Cancer 30B: 191-195. 1994

32. Nagatsuka H, Han PP, Tsujigiwa H, Siar CH, Gunduz M, Sugahara T, Sasaki A, Nakajima M, Naomoto Y and Nagai N. Heparanase gene and protein expression in ameloblastoma: possible role in local invasion of tumor cells. Oral Oncol 41: 542-548, 2005

33. Nagatsuka H, Shin H-I, Park H-K, Ishiwari Y, Kuroda K, Nosaka Y, Song H, Qin C-L, Zhang S-Q, Nakano K, Chigono Y, Tsujigiwa H, Takagi T and Nagai N. Immunohistochemical study of cytokeratin patterns in follicular and plexiform ameloblastoma. J Hard Tissue Biol 4: 50-56, 1995

34. Nagatsuka H, Siar CH, Kitamura Y, Tsujigiwa H, Lee Y-J, Gunduz M, Tamamura R, Komori T, Lefevre M and Nagai N. Gene expression of matrix proteins in Cbfa1-knockout mice. J Hard Tissue Biol 13: 37-46, 2004

35. Nagatsuka H, Siar CH, Nakano K, Tsujigiwa H, Gunduz M, Choufuku H, Lee Y-J, Naito I, Sado Y and Nagai N. 
Keisuke Nakano et al.: Immunohistochemistry of Odontogenic Neoplasms

Differential expression of collagen IV $\alpha 1$ to $\alpha 6$ chains in basement membranes of benign and malignant odontogenic tumors. Virch Arch 441: 392-399, 2002

36. Nakano K, Siar CH, Nagai N, Naito I, Sado Y, Nagatsuka H, Hor C, Kuroda K, Tsujigiwa H and Gunduz M. Distribution of besement membrane type IV collagen a chains in ameloblastoma: an immunofluoressence study. J Oral Pathol Med 31: 494-499, 2002

37. Nakano S, Iyama K, Ogawa M, Yoshioka H, Sado Y, Oohashi $\mathrm{T}$ and Ninomiya Y. Differential tissular expression and localization of type IV collagen alpha1(IV), alpha2(IV), alpha5(IV), and alpha6)IV) chains and their mRNA in normal breast and in benign and malignant breast tumors. Lab Invest 79: 281-292, 1999

38. Nakano K, Chelvanayagam P, Born K, Siar CH, Ng KH, Nagatsuka $\mathrm{H}$ and Kawakami T. A study of recurrent giant odontogenic myxoma of the mandible with immunohistochemical examination of Notch. Oral Med Pathol 12: 53-56, 2008

39. Nakano K, Siar CH, Tsujigiwa H, Nagatsuka H, Nagai N and Kawakami T. Notch signaling in ameloblastic neoplasms. Eur J Med Res 13: in Press, 2008

40. Ng KH and Siar CH. Peripheral ameloblatoma with clear cell differentiation. Oral Surg 70: 210-213, 1990

41. Ninomiya Y, Kagawa M, Iyama K, Naito I, Kishiro Y, Seyer JM, Sugimoto M, Oohashi T and Sado Y. Differential expression of two basement membrane collagen genes, COL 4 A 6 and COL 4A5, demonstrated by immunofluorescence staining using peptide-specific monoclonal antibodies. J Cell Biol 130: 1219-1229, 1995

42. Ono A, Tsukamoto G, Nagatsuka H, Yoshihama Y, Rivera RS, Katsurano M, Yao M, Sasaki A. An immunohistochemical evaluation of BMP-2, -4, osteopontin, osteocalcin and PCNA between ossifying fibromas of the jaws and peripheral cemento-ossifying fibromas on the gingiva. Oral Oncology. 43: 339-344, 2007

43. Parish CR, Freeman C and Hulett MD. Heparanase: a key enzyme involved in cell invasion. Biocem Biophys Acta 1471: M99-108, 2001

44. Philipsen HP, Ormiston IW and Reichart PA. The desmo and osteoplastic ameloblastoma. Histologic valiant or clinicopathoilogic entity? Case reports. Int J Oral Maxillofac Surg 21: 352-357, 1992

45. Reichart PA, Philipsen HP and Sonner S. Ameloblastoma: biological profile of 3677 cases. Eur J Cancer B Oral Oncol 31B: 86-99, 1995

46. Sado Y, Kagawa M, Kishiro Y, Sugihara K, Naito I, Seyer JM, Sugimoto M and Oohashi T. Establishment by the rat lymphnode method of epitope-defiened monoclonal antibodies recognizing the six different a chains of human type IV collagen. Histochem Cell Biol 104: 267-275, 1995
47. Sado Y, Kagawa M, Naito I, Ueki Y, Seki T, Momota R, Oohashi $\mathrm{T}$ and Ninomiya Y. Organization and expression of basement membrane collagen IV genes and their roles in human disorders. J Biochem 123: 767-776, 1998

48. Sathi GS, Han PP, Tamamura R, Nagatsuka H, Hu H, Katase $\mathrm{N}$, Nagai N. Immunolocalization of cell signaling molecules in the granular cell ameloblastoma. J Oral Pathol Med 36: 609-614, 2007

49.Siar $\mathrm{CH}$ and $\mathrm{Ng} \mathrm{KH}$. Calcifying and keratinizing ameloblastoma of the maxilla. J Laryngol Otol 105: 971-972, 1991

50. Siar CH, Ng KH, Ariff Z, Muraki E, Shimizu T, Tsujigiwa H, Nagatsuka H, Nagai $\mathrm{N}$ and Kawakami T. A case report of ameloblastoma of the mandible with examination of Notch signaling. Oral Med Pathol 11: 35-39, 2006

51. Slater LJ. Odontogenic sarcomas and carcinosarcomas. Semin Diagn Patholo 16: 325-332, 1999

52. Takei N, Kawakami T, Kawai T, Yoshikawa Y and Eda. Expression of TGF- $\beta$ in the course of BMP-induced hetrotopic osteogenesis. J Hard Tissur Biol 6: 64-69, 1997

53. Tamamura R, Nagatsuka H, Lee Y-J, Xiao J, Naito I, Sado Y, Kawakami T and Nagai N. Distribution of collagen type IV á1-6 chains and MMP in carcinogenesis in oral squamous cell carcinoma. J Hard Tissue Biol 13: 117-123, 2004

54. Tamamura R, Nagatsuka H, Lee Y-J, Xiao J, Naito I, Sado Y, Kawakami T and Nagai N. Distribution of collagen type IV $\alpha 1-6$ chains and MMP in oral squamous cell carcinoma. J Hard Tissue Biol 13: 125-130, 2004

55. Tanaka K, Iyama K, Kitaoka M, Ninomiya Y, Oohashi T, Sado $\mathrm{Y}$ and Ono T. Differential expression of alpha 1 (IV), alpha 2 (IV), alpha 5 (IV) and alpha 6 (IV) collagen chains in the basement membrane of basal cell carcinoma. Histochem J 29: 563-570, 1997

56. Tezuka K, Yasuda M, Watanabe N, Morimura N, Kuroda K, Miyatani S and Hozumi N. Stimulation of osteoblasatic cell differentiation by Notch. J Bone Miner Res 17: 231-239, 2002

57. Thesleff I, Barrach HJ, Foidart JM, Vaheri A, Pratt RM and Martin GR. Changes in the distribution of typr IV collagen, laminin, proteoglycan, and fibronectin during mouse tooth development. Dev Biol 81: 182-192, 1981

58. Thesleff I and Ekblom P. Disyribution of keratin and laminin in amelkoblastoma. Comparisonwith developing tooth and epidermoid carcinoma. J Oral Pathol 13: 85-96, 1984

59. Tomich CE (1999) Benign mixed odontogenic tumors. Semin Diagn Pathol 16: 308-316.

60. Tsujigiwa H, Nagatsuka H, Han PP, Gunduz M, Siar CH, Oida $\mathrm{S}$ and Nagai. Analysis of amelogenin gene (AMGX, AMGY) expression in ameloblastoma. Oral Oncol 41: 843-850, 2005

61. Yurchenco PD and O’Rear JJ. Basal lamina assembly. Curr Opin Cell Biol 6: 674-681, 1994 
J.Hard Tissue Biology Vol. 17(3):79-90. 2008

62. Zagouras P, Stifani S, Blaumueller CM, Carcangiu ML and Artavanis-Tsakonas S. Alterations in Notch signaling in neoplastic lesions of the human cervix. Proc Natl Acad Sci USA 92: 6414-6418, 1995

63. Zcharia E, Metzger S, Chajek-Shaul T, Aingorn H, Elkin M,
Friedmann Y, Weinstein T, Li JP, Lindahl U and Vlodavsky I. Transgenic expression of mammalian heparanase uncovers physiological functions of heparansulfate in tissue morphogenesis, vascularization, and feeding behavior. FASEB J 18: 252-263, 2004 